\title{
A VIRTUAL BRAIN FOR STEREOTACTIC PLANNING AND SUPPORTING NEUROSURGERY
}

\author{
Zigang Wang, Zesheng Tang \\ Tsinghua University, P.R. China \\ Tianmiao Wang, Mengdong Chen, Da Liu \\ Beijing Univ of Aeronautics and Astronautics, China \\ Zengmin Tian \\ Navy Central Hospital, China
}

\begin{abstract}
Visualization has becoming a powerful assistive technology for neurosurgery. This paper introduces a system for stereotactic neurosurgical planning and support. Using visualization technology the system reconstructs and displays a 3D model of the interior structure of the patient's brain. Thus the surgeons can plan for surgery using a computer model. Marker registration is used to create the mapping between the patient's head and the brain model reconstructed in the computer. During the operation a robot arm is used as a navigator to locate the pre-defined incision site and the orientation of incision route. When the robot arm locates at the pre-defined site on the patient's head, it is fixed. Various medical instruments can be installed on the tip of the robot arm. The surgeon can insert a medical instrument into the pre-defined site of the patient's head, and the surgery can be implemented successfully with the help of this system. Using a virtual environment his system can also be used to teach and train new surgeons.
\end{abstract}

\section{Introduction}

With the rapid progress of computer hardware and software, visualization technology is becoming a powerful method to view the data of scientific computing, including measured data in the medical field. More and more visualization systems for medical diagnosis and surgery planning are being used in hospitals $[1,2,3,5,6]$. New developments in volume data acquisition, such as the CT, MRI, PET, SPECT etc., provide surgeons with precise and detailed information about the patient's inner body. With this information the patient's 3D anatomical and pathological structures can be reconstructed using visualization technology, allowing surgeons to clearly understand the exact character of the operative site and how it relates to adjacent critical anatomical structures. The removal of some pathology can become safer and more accurate.

The conventional neurosurgery is based on opening the patient's skull to expose the brain organ for operation. In that process, a judgement of the location of the pathological tissue depends on the surgeon's understanding of the relation between the 2D images from the CT, MRI, etc, and the physical 3D structure of the patient's head. This method also heightens the pain and risk of infection, and can lead to a longer convalescence following the surgery.

To overcome these shortcomings, more and more stereotactic neurosurgeries are now used in hospitals $[2,3,5,6,9,10]$. With the stereotactic method, the surgeon only needs to bore a hole on the patient's skull and insert the minimally invasive instruments into the brain through the hole. When the instrument reaches the pathological tissue, surgeon can use it to remove the tissue or inject some material into the brain. Obviously this method reduces patient's pain, decreases the risk of infection, and brings the patient shorter recovery time. 
Ryo Yoshida [2] developed a planning and supporting system: CliPSS. A frame fixed on the pattient's head is used to establish the exact location of the tissue and the instrument. Unfortunately, the frame limits the access of the instrument and causes discomfort and inconvenience to the patients. Birgit Westermann [1], Grimson [5], and Tebo [6] use a 3D digitizer and camera to accurately locate the probe. Because of the cost and limitations of the digitizer, these systems cannot adequately support the neurosurgery. Philippe [9] used the 3D atlas to provide the neurosurgeon with missing information. This method can make the visualization and surgery planning more convenient, but the system still uses a frame to locate the position. Lisa [10] used the AVW Toolkit to develop a visualization tool for "line-of-sight" stereotactic neurosurgical navigation. The system provides the surgeons with a planning tool, but it doesn't support the neurosurgery operation.

A new robot-arm-aided neurosurgery planning and support system is introduced in this paper. This system combines visualization technology with a robot arm to realize frameless stereotactic neurosurgery planning and support. Discarding the frame, marker registration is used to make precise mapping of the patient's head, the robot arm, and the model of the patient's brain in the computer. The robot arm is used as a navigator to direct the surgery planning, and also as an instrument platform to assist surgeons to accomplish the stereotactic neurosurgery.

\section{Stereotactic Neurosurgery Planning and Support System}

The surgery planning and support system is a platform that provides surgeons with tools to make a pre-operative surgerical plan and a navigator to locate the correct location of the incisive site, even the orientation of the instrument as well as the bore depth during the surgery. It is also a platform for inserting the medical instrument to do the operation.

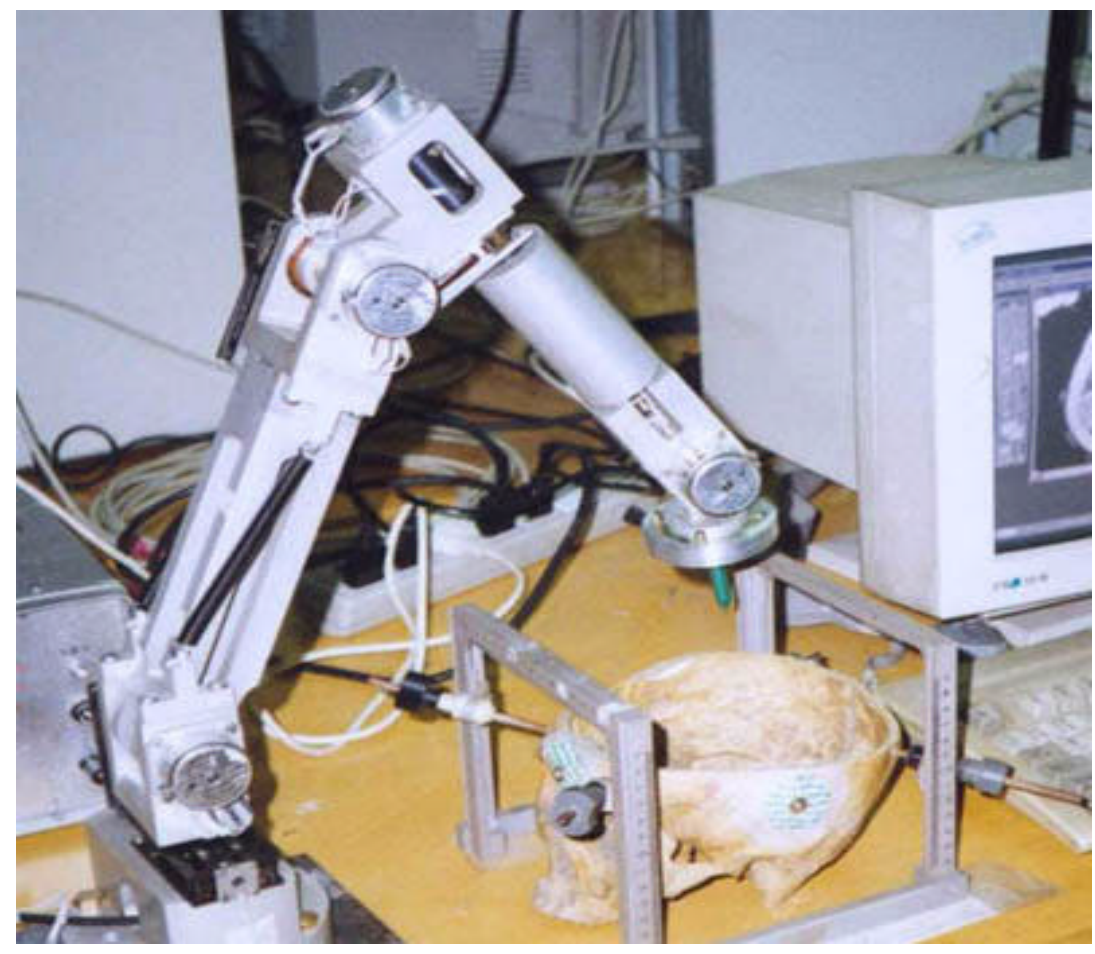

Fig. 1. The powerless 5-joint digital arm. 
The system is developed on a PC workstation and is made up of a software module and a hardware module. The software module is programmed in $\mathrm{C}++$. Its structure and main functions are described in Fig. 2. The hardware module is uses a PC framework and a powerless 5-joint mechanical digital arm (See Fig. 1) that we refer to as the robot arm in following sections. The motion status of the arm can be calculated based on the joint angle values input into the computer.

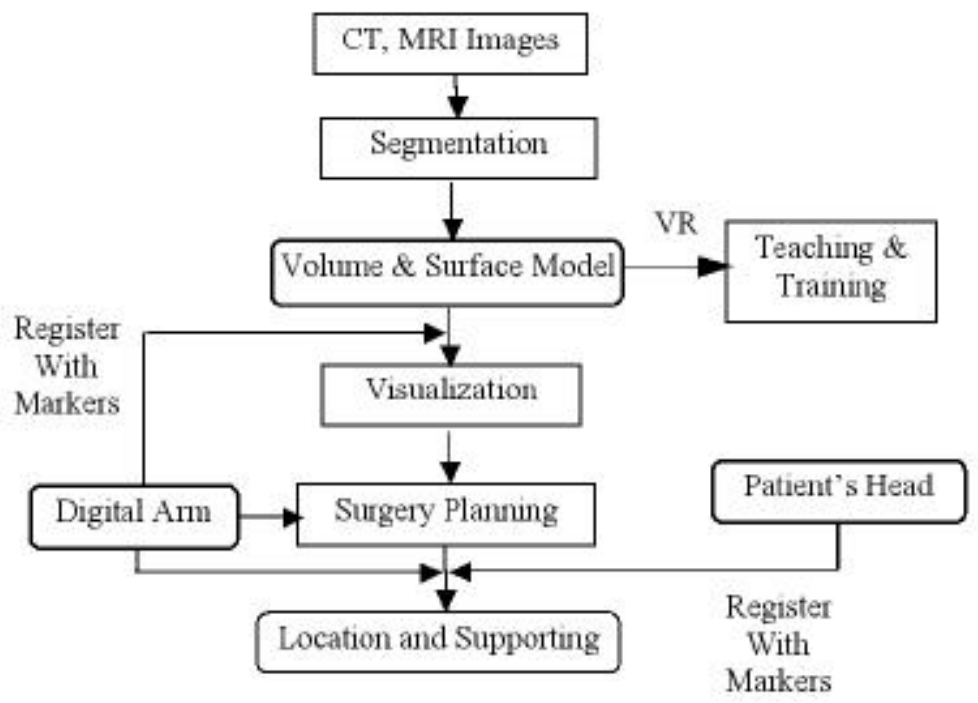

Fig. 2. Flow Chart of the Surgery Planning and Supporting System

\subsection{SEGMENTATION AND DEMARCATION}

This system uses the CT and MRI images of the patient's head as the original data to reconstruct the 3D model of the patient's brain. After the CT and MRI images are input into the system, the critical anatomical tissues and pathological regions must be distinguished from the adjacent tissues. Some anatomical tissues and pathological regions are difficult to distinguish from the adjacent region in the images. Only trained eyes can distinguish them. In the identification of the pathological tissues, a surgeon's judgment and decision making are necessary. In order to get the correct segmentation, the system provides not only some automatic segmentation function to get the edges of certain tissues, but also the interactive tools to help the surgeon to distinguish the pathological regions and critical tissues manually.

The gray levels of CT and MRI images are almost identical in some regions. To reduce the surgeon's work, we developed a new region border extraction algorithm [4] to automatically extract and display the boundaries of the tissues. This algorithm is based on the scan-line filling method, and it can efficiently extract the boundaries of four-connected raster regions. All boundaries of the regions (inner or outer) can be generated in one scanning process that is independent of the region's form (Fig. 3). Chain code, a border representation well suited for a raster image, is used to represent the results of the extracted boundaries in the form of closed contours. 


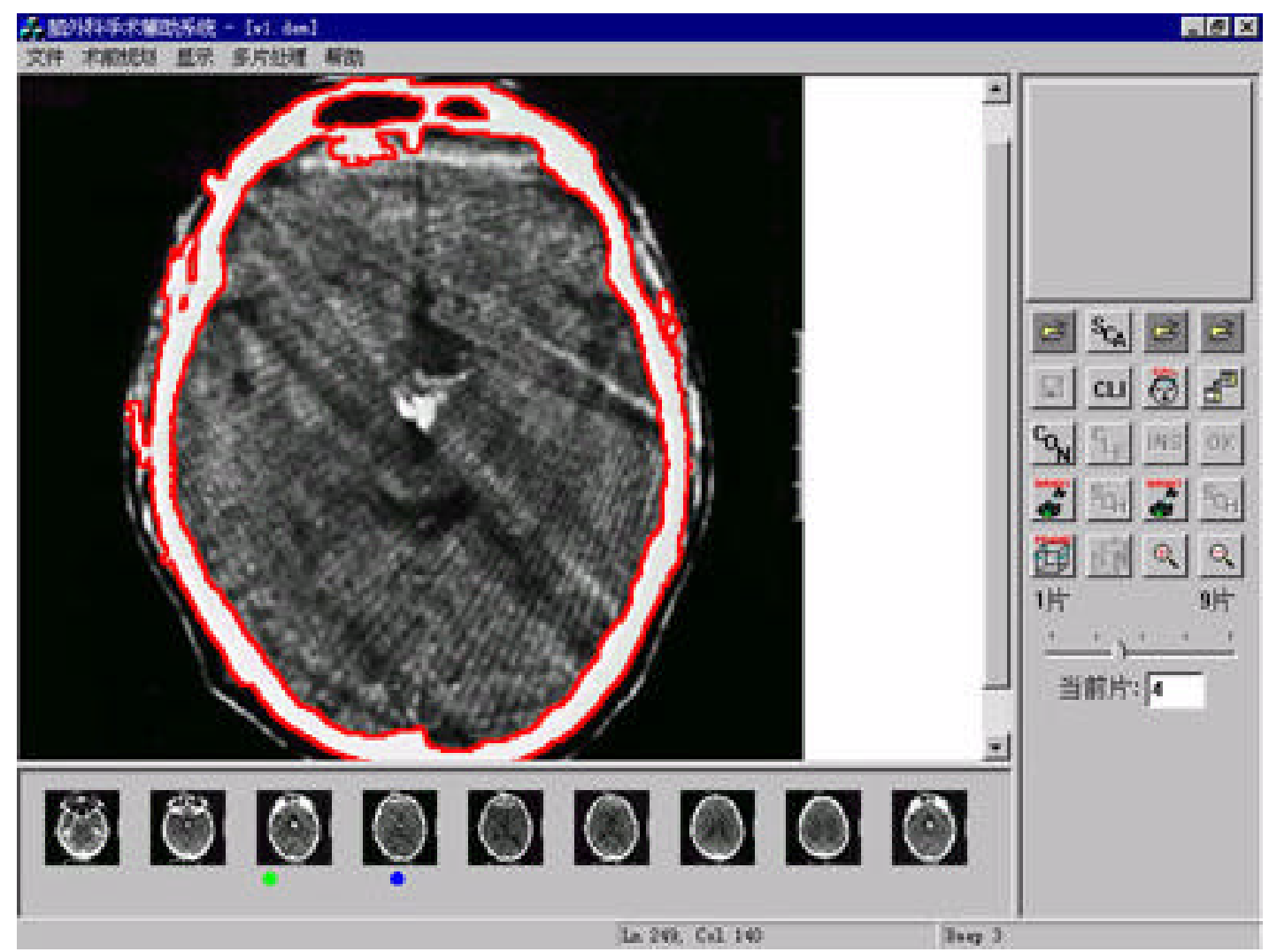

Fig. 3. The red contour is extracted by the new algorithm automatically.

\subsection{RECONSTRUCTION AND VISUALIZATION}

After segmentation and demarcation, the surface and volume model of the specific anatomical and pathological tissues can be reconstructed by the system. The algorithm for tiling the triangles from planar contours is one of the choices [7,8] for the surface model. In the algorithm the correspondence of the contours is first confirmed and then all contours are tiled using triangles. The ultimate result is a model of the surface made up of triangles reconstructed from the images (it is called the model in the following sections). The volume model is a standard voxel set. The surface model is rendered by conventional illumination algorithms, and the volume model can be rendered by volume rendering.

In the practical application of this system, the CT and MRI images are input $0.5 \sim 1$ hours before the operation, so the images can reflect the recent brain status of the patient. The model is an accurate representation of the brain, allowing surgeons to see the patient's critical and pathelogical tissues accurately on a computer screen. (Fig. 4). 


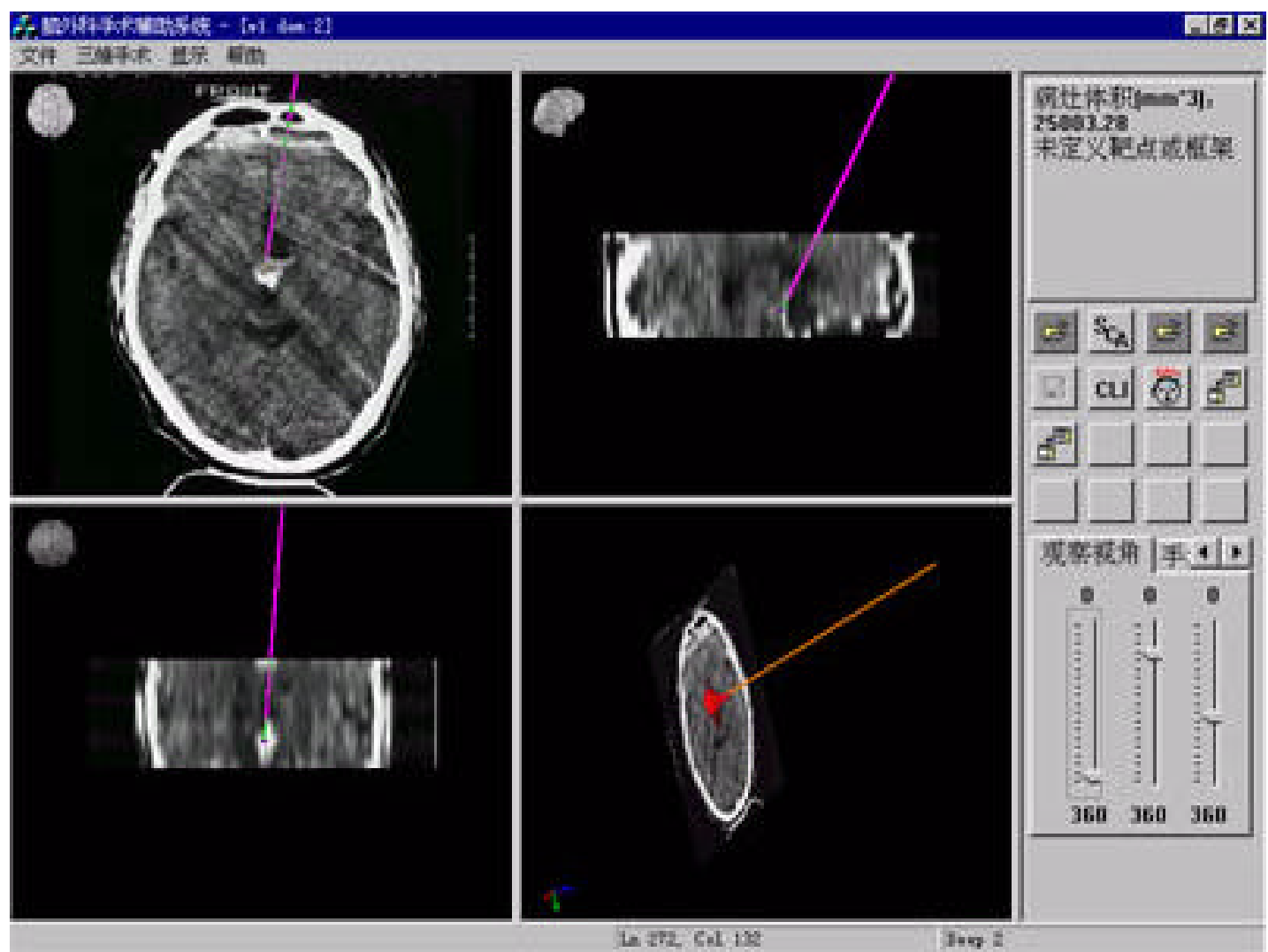

Fig. 4. The surgery planning interface of the system.

The red model at right bottom is the visualization of $3 D$ surface model of the pathology, which is defined by the surgeon. The purple line is the defined incisive route.

\subsection{SURGERY PLANNING}

Since the model in the computer is reconstructed from instant CT and MRI images of the patient, the model can reflect the current status of the brain. Surgeons can manipulate the model to simulate the operation which will be done on the patient.

As for the stereotactic surgery, the correct location of the incisive site and the orientation and the depth of the medical instrument are the key issues. The system provides surgeons with some metrical information such as the volume of pathological tissue and the long axis orientation. The information helps the surgeon determine the location of the incision site and the route by which the surgical instruments should be inserted into the brain. With the visualization model, the surgeons can define the route of the incision to avoid severing critical vessels or nerves. The system will display the surgerical plan designed by the surgeon repeatedly until it is complete and satisfactory.

\subsection{ROBOT ARM REGISTRATION}

We define three kinds of spaces: the space of the model constructed in the computer, called the model space; the space of the patient, which is called the patient space; and the local space of the robot arm, which is called the robot space. A successful operation relies on the correct registration of these three spaces. 
To make the registration the direct and accurate method is to find out the transform relations between their coordinate systems. Since all the models are generated from the same patient, the relations among different models can be determined by a rigid transformation, including rotation, scaling, and translation.

The coordinate system of the model space can be defined naturally, but it is difficult to accurately depict the patient's local coordinate system naturally. Thus we must attach an external coordinate system to the patient head. In our system a frameless reference-marker system is used instead of a cumbersome metal frame.

The marker method is easy to perform and brings a little discomfort to the patient. Four or more markers are fixed (possibly with glue) to the patient's head before the operation (Fig. 5), then the patient is scanned by a CT or an MR scanner. Attached on the skin, the markers are visible in the CT or MRI images (Fig. 6). The system can identify them and their tips easily.

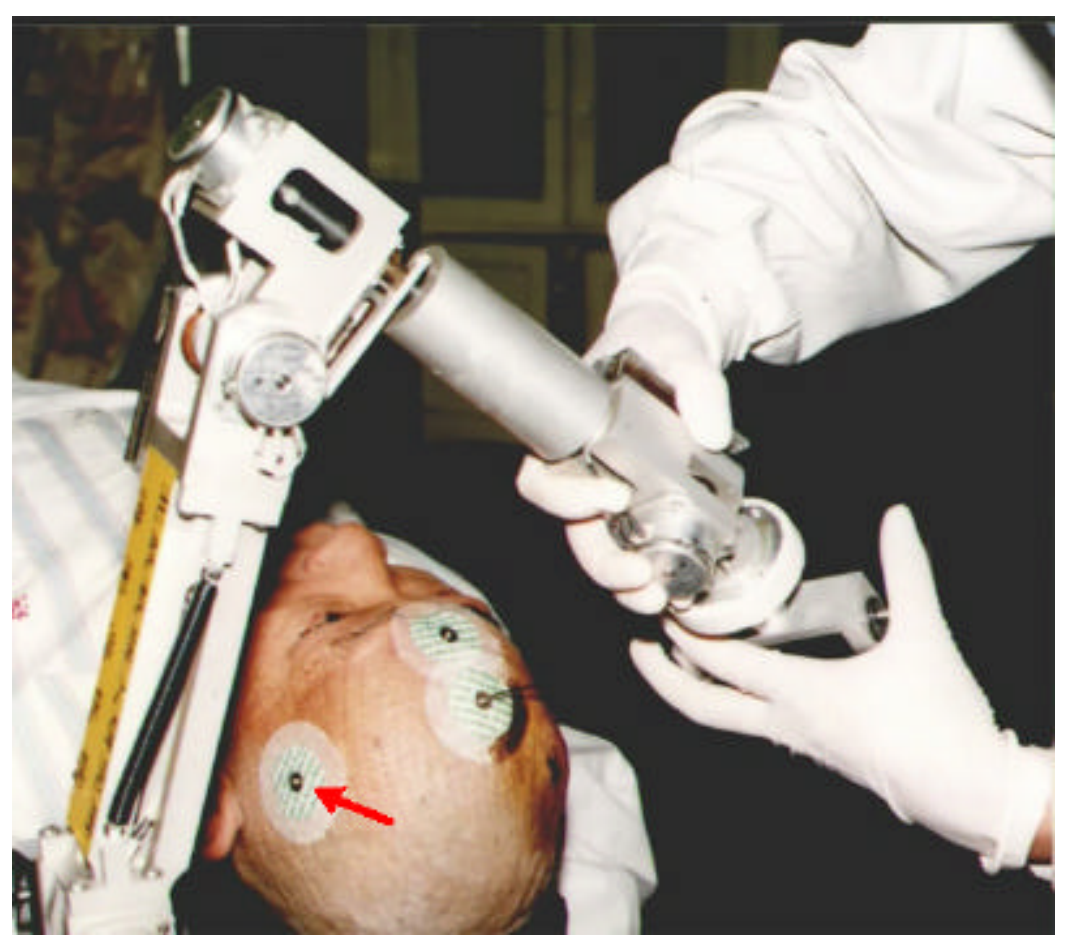

Fig. 5. The marker which is attached on the patient head (Pointed by he red arrow) 


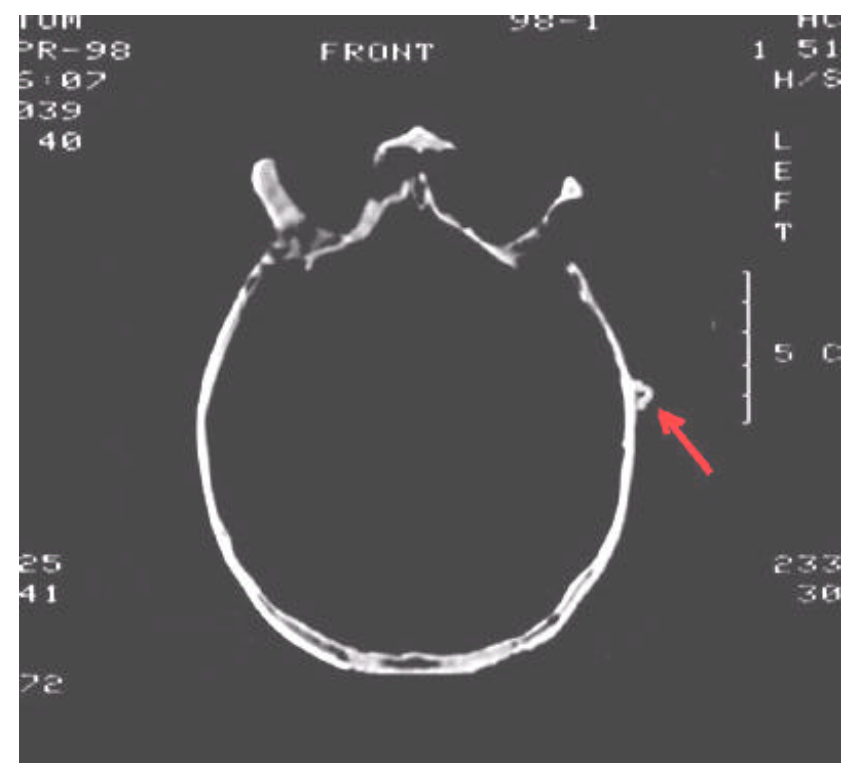

Fig. 6. The mapped marker in the CT image (Pointed by the red arrow)

To set up the patient's coordinate system we select four markers to define a non-orthogonal coordinate system. (Any three of them cannot be on the same straight line, and the four of them cannot be on the same plane) One marker $M_{o}$ is selected as the origin of this reference system, and another maker $M_{m l}$ is selected to form the X axis. The $M_{m l}$ 's coordinate in this reference system is $(1.0,0.0,0.0)$. Similarly we use the other two markers $M_{m 2}, M_{m 3}$ to form the Y-axis and the Z-axis. Then each point of the patient's head $M_{p}$ can be considered as a triple $\left(x_{p}, y_{p}, z_{p}\right)$ where

$$
\overrightarrow{M_{o} M_{p}}=x_{p} \cdot \overrightarrow{M_{o} M_{m 1}}+y_{p} \cdot \overrightarrow{M_{o} M_{m 2}}+z_{p} \cdot \overrightarrow{M_{o} M_{3}}
$$

When the corresponding markers are identified in the model, we can get their coordinate values in the model's reference system as shown in the following table:

\begin{tabular}{|c|c|c|}
\hline & Patient Space & Model Space \\
\hline$M_{o}$ & $(0.0,0.0,0.0)$ & $\left(x_{o}, y_{o}, z_{o}\right)$ \\
\hline$M_{m 1}$ & $(1.0,0.0,0.0)$ & $\left(x_{m 1}, y_{m 1}, z_{m 1}\right)$ \\
\hline$M_{m 2}$ & $(0.0,1.0,0.0)$ & $\left(x_{m 2}, y_{m 2}, z_{m 2}\right)$ \\
\hline$M_{m 3}$ & $(0.0,0.0,1.0)$ & $\left(x_{m 3}, y_{m 3}, z_{m 3}\right)$ \\
\hline
\end{tabular}

The transform matrix from patient reference system to model reference system is described by equation (2):

$$
T_{1}=\left[\begin{array}{cccc}
x_{m 1}-x_{o} & x_{m 2}-x_{o} & x_{m 3}-x_{o} & x_{o} \\
y_{m 1}-y_{o} & y_{m 2}-y_{o} & y_{m 3}-y_{o} & y_{o} \\
z_{m 1}-z_{o} & z_{m 2}-z_{o} & z_{m 3}-z_{o} & z_{o} \\
0 & 0 & 0 & 1
\end{array}\right]
$$

Then each point in the patient reference system $p_{\text {patient }}$ can be mapped into the model system by equation (3). 


$$
p_{\text {model }}=T_{1} \cdot p_{\text {patient }} \quad p_{\text {patient }}=T_{1}^{-1} \cdot p_{\text {model }}
$$

After the surgeon fixes the patient's head on the operating table, the robot arm is used to touch the pre-defined four markers on the patient's head. When robot arm's tip touches each marker, the surgeon sends a message to the system. Then the angle data from each joint is transferred into the computer. By calculating the joints' rotate angle, the system can get the tip location from the robot arm's local reference system. Using a similar transform matrix to eqaution (2), we get the transform matrix $\mathrm{T}_{2}$ from the robot reference system to patient system, then each point in the patient reference system $p_{\text {patient }}$ can be mapped into the robot reference system by equation (4).

Combining equatiosn (3) and (4), the link between robot reference system and model reference system is also established. The transform equation is equation (5).

$$
\begin{array}{ll}
p_{\text {robot }}=T_{2} \cdot p_{\text {patient }} \quad p_{\text {patient }}=T_{2}^{-1} \cdot p_{\text {robot }} \\
p_{\text {robot }}=\left(T_{2} \cdot T_{1}^{-1}\right) \cdot p_{\text {model }} & p_{\text {model }}=\left(T_{1} \cdot T_{2}^{-1}\right) \cdot p_{\text {probot }}
\end{array}
$$

Thus the links among the patient, the model, and the robot arm have been created. Following this, any motion of the robot arm will be mapped into the model reference system in computer. Any location of the model in the computer can be considered as the actual location on the patient's head (Fig. 7).

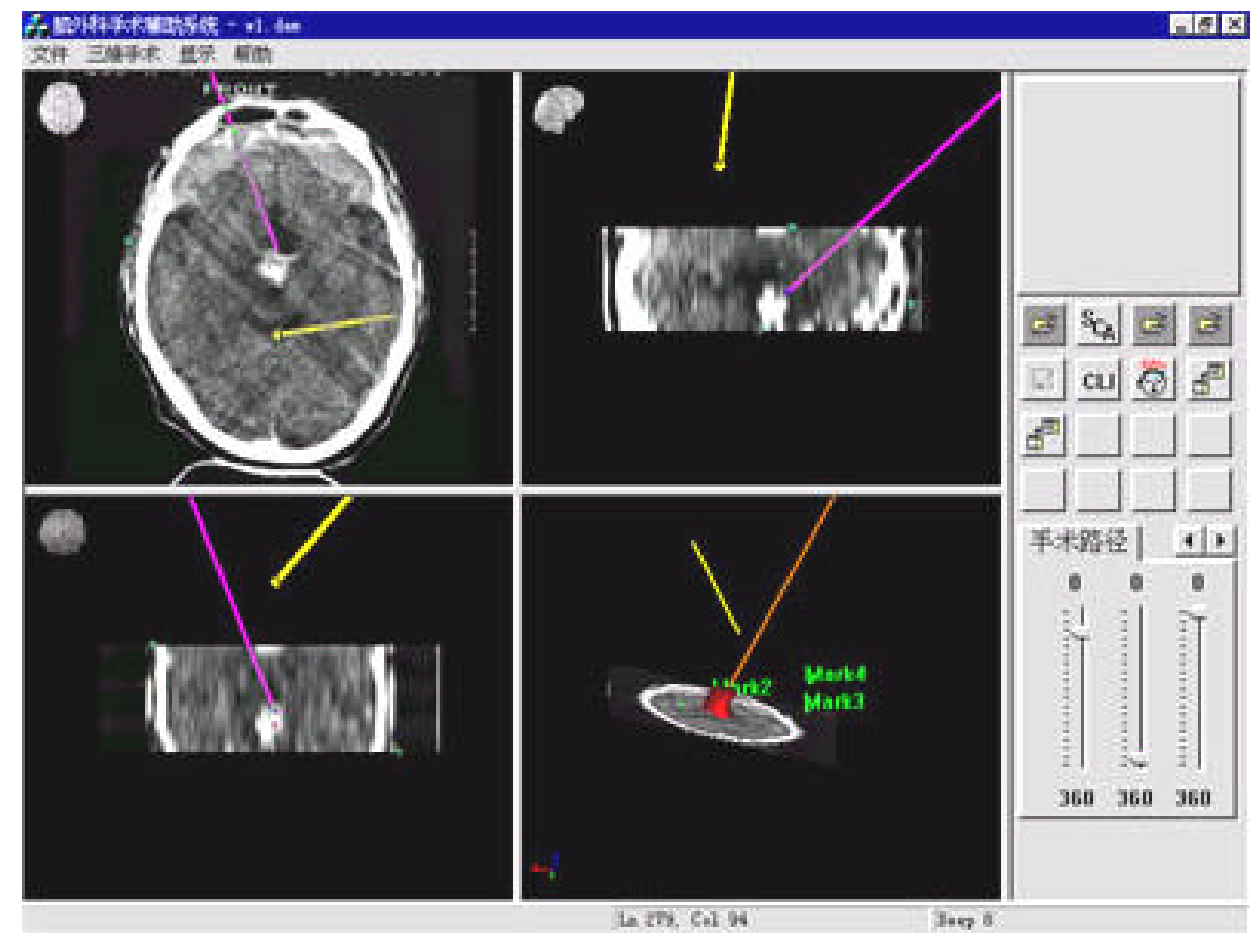

Fig. 7. The robot mapped on the model (The yellow line is the front arm of the robot arm) 


\subsection{SURGERY SUPPORT}

Since the robot arm and patient's location can be mapped to the model in the computer, when the surgeon moves the robot arm around the patient's head, the correct relative location can also be displayed on the screen. When the mapped robot arm is located on the pre-designed incision site of the model in the computer, the actual location of the robot arm is exactly at the site on the patient's head where the surgeon will bore the hole. The surgeon can modify the arm's orientation to match the pre-designed orientation exactly (Fig. 7). Now the system can calculate the depth between the recent location and the destination, providing the surgeon with the precise information.

The robot arm is not only a navigator but also a medical instrument platform. When the arm is fixed at a specific location, the system can lock the arm's joint to prevent it from moving. Then the surgeon can install various medical instruments (Fig. 8) on the tip of the arm for different surgical tasks.

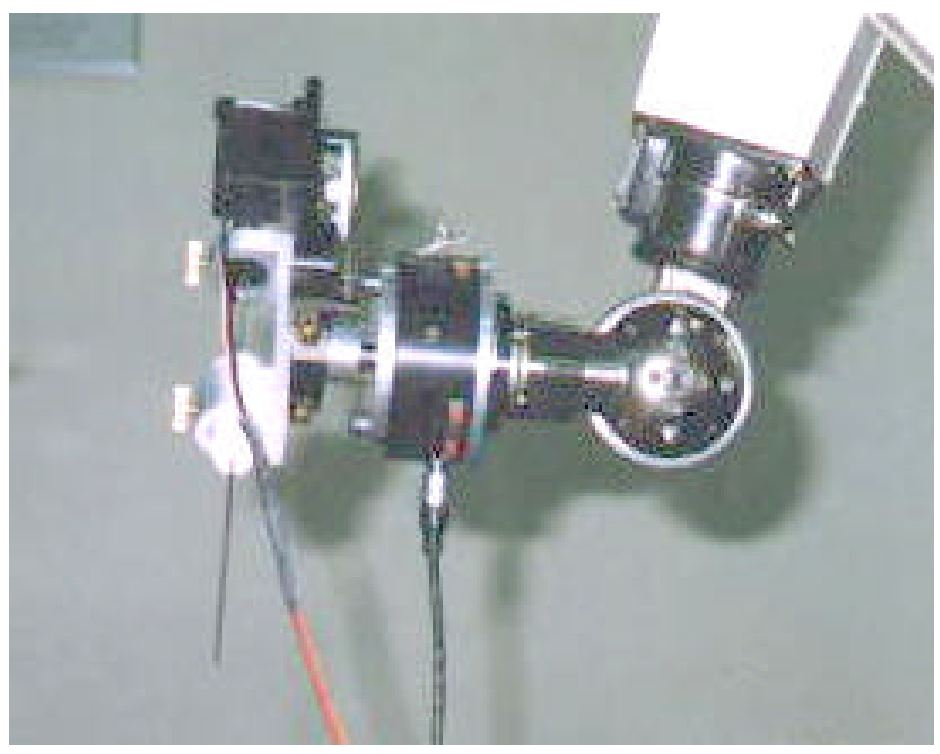

Fig. 8. The surgical instrument installed on the tip of the digital arm.

Sometimes the surgeons needs to inject radioactive material into the patient's head to kill the tumor. Conventionally this dangerous operation is done by the surgeons themselves or by their assistants. To prevent the surgeons from the risk of exposure to the radiation, the system allows the robot to do the injection by remote control.

\section{Experimental Results}

As a stereotactic surgery support system, the accuracy of the system is the most important criterion for evaluating its practicality. To test the accuracy of the whole system, several trials were done independently by a group of people. Four markers were fixed on the surface of a skull, and three additional markers (called targets in the following sections) were fixed in the interior of the skull. When the tip of the mapped robot arm reached these targets in the model, the distance between the tip of the robot arm and the actual marker was calculated. That distance was considered as the error of the system. The statistical results are shown in the Table 1. 


\begin{tabular}{|c|c|c|c|}
\hline $\begin{array}{c}\text { Error } \\
\text { Statistic }\end{array}$ & $\begin{array}{c}\text { Target1 } \\
(\mathbf{m m})\end{array}$ & $\begin{array}{c}\text { Target2 } \\
(\mathbf{m m})\end{array}$ & $\begin{array}{c}\text { Target3 } \\
(\mathbf{m m})\end{array}$ \\
\hline Mean & 2.0870 & 2.1935 & 1.9907 \\
\hline $\begin{array}{c}\text { Standard } \\
\text { Deviation }\end{array}$ & 0.7492 & 0.8153 & 0.8075 \\
\hline $\begin{array}{c}\text { Maximu } \\
\text { m }\end{array}$ & 2.57 & 3.5 & 3.3 \\
\hline Minimum & 0.92 & 1.07 & 1.08 \\
\hline $\begin{array}{c}\text { Number } \\
\text { of the } \\
\text { Trial }\end{array}$ & 30 & 30 & 30 \\
\hline
\end{tabular}

Table 1. The error statistic of the neurosurgery planning and supporting system.

The error of the system might have been caused by the inaccuracy of the angle sensors installed on the joints of the robot arm. The error of the A/D board could also cause the deviation of the actual position from the calculated position of the computer. Since the CT image is the fused image of the skull whose precision is $2 \mathrm{~mm}$, two positions in the same image can have different depths and thus also cause errors.

Using this system, more than fifteen stereotactic frameless nerosurgery operations have been done (Fig. 5 shows one of these operations). The locations of the probe and the other medical instruments are accurate. All operations on the patients aged from eight to fifty were successful in that after the operations, their symptoms were relieved or disappeared.

\section{Conclusion and Future Work}

This system was developed for stereotactic neurosurgery planning and support. Given the patient's CT and MRI images, the system reconstructs the 3D model of the critical anatomical tissues and the pathological structure. Using visualization technology, the system lets the surgeons observe the inner structure of the patient's head. The surgeon can make a pre-operative plan using the model. The marker registration method is used in the system to construct the link between the patient, the model, and the robot arm. In this way the robot arm can be used to locate a pre-defined incisive site with correct orientation. The robot arm is also used as a platform in which various medical instruments can be installed. After the robot arm is moved to the pre-defined site, it is locked. Surgeons can then use the instrument installed on the robot arm in the operation.

Our future work is to extend this system to a teaching and training system in a virtual reality environment. Using the volume atlas and labeling each section of the brain, the 3D virtual brain can be reconstructed in the computer. An ordinary skull model can be used instead of the patient head. When the tip of the robot arm is moving around in the interior of the skull, the user can explore the interior structure of the brain. It will be a teaching and training system in VR for stereotactic neurosurgery. If the $3 \mathrm{D}$ virtual brain is reconstructed with the real image of the patient, this system can also be used to show structures around the tip of the medical instrument in the interior of the brain in the VR environment during stereotactic neurosurgery. 


\section{REFERENCES}

[1] Birgit Westermann, Rolf Hauser, "Non-invasive 3-D Patient Registration for Image-guided Skull Base Surgery", Computers \& Graphics, vol. 20, no. 6, 1996, pp.793-799

[2] Ryo Yoshida, Tatsuo Miyazawa, et al. "Clinical Planning Support System-CliPSS", IEEE Computer and Application, November 1993, pp.76-84.

[3] Stephen T. C. Wong, Robert C. Knowlton, et al. "Multimodal Image Fusion for Noninvasive Epilepsy Surgery Planning”, IEEE Computer and Application, January 1996, pp. 30-38.

[4] Zigang Wang and Zesheng Tang, "A Boundary Extraction Algorithm for Raster Image Region", Proceedings of CAD \& Graphics'97-Fifth International Conference on CAD \& CG, December 2-5, 1997, Shenzhen, China, pp.190-193.

[5] W. E. L. Grimson, G. J. White, et al. "An Automatic Registration Method for Frameless Stereotaxy, Image Guided Surgery, and Enhanced Reality Visualization”, IEEE Trans. On Medical Imaging, vol.15, no. 2, April 1996, pp. 129-140.

[6] Scot A. Tebo, Donald A. Leopold, et al. "An optical 3D Digitizer for Frameless Stereotactic Surgery", IEEE Computer Graphics and Application, January 1996, pp. 55-64.

[7] Gill Barequet and Micha Sharir, "Piecewise-Linear Interpolation between Polygonal Slices", Computer Vision and Image Understanding, vol.63, no.2, March 1996, pp. 251-272.

[8] David Meyers, Shelley Skinner and Kenneth Sloan, "Surface from contours", ACM Trans. on Graphics, vol. 11, no.3, July 1992, pp.228-258.

[9] Philippe St-Jean, Reza Kasrai and Diego Clonda, et al. "Interactive 3-dimensional visualization tools for stereotactic atlas-based functional neurosurgery", SPIE Conference on Image Display, vol.3335, February 1998, pp.198-207.

[10] Lisa M. Bates, Dennis P. Hanson, Bruce A. Kall, et al. "Implementation of an Oblique Sectioning Visualization Tool for "Line-Of-Sight" Stereotactic Neurosurgical Navigation Using the AVW Toolkit", SPIE Conference on Image Display, vol.3335, February 1998, pp. 219-227.

\section{BIOGRAPHIES}

Zigang Wang is a Ph.D. candidate of the Department of Computer Science and Technology at Tsinghua University, China. He received his B.S. and M.S. degrees from Shandong University, China. His major is in scientific visualization and computer graphics. His current research interests include computer graphics, VR, medical visualization, and volume deformation.

Zesheng Tang is a professor of the Department of Computer Science and Technology at Tsinghua University, China. He is also the president of the China Computer Federation (CCF), an IEEE senior member, and an ACM member. He has published 4 books and more than 100 papers. His current research interests include scientific visualization, computer graphics, virtual reality, and computational geometry.

\section{Contact information:}

Zesheng Tang

Department of Computer Science and Technology,

Tsinghua University,

Beijing, China

Phone: +86-010-62782052

Email: mailto:ztang@mail.tsinghua.edu.cn 